\title{
Desandar caminos, reconstruir saberes. Una aproximación arqueológica a la zona serrana y periserrana del partido de Tandil
}

\section{(1) Selene Arislur*}

\author{
Fecha de defensa: 9 de Octubre de 2017 \\ Director: Dr. Damián Bozzuto \\ Co-directora: Dra. Natalia Mazzia \\ Jurados: Dras. Paula Escosteguy y Magdalena \\ Frère
}

A partir de esta tesis buscamos profundizar y ampliar las discusiones sobre el pasado prehispánico del partido de Tandil, porción central del sistema serrano Tandilia, centro-este de la provincia de Buenos Aires. Abordamos la problemática desde una perspectiva reflexiva y relacional, ya que comprendemos que los procesos de producción de conocimiento están mediados por situaciones de encuentro y negociación de relaciones sociales (Achilli, 2005).

Producto de sistemáticas investigaciones arqueológicas, conocemos que el paisaje del sistema serrano de Tandilia, ubicado en la región pampeana bonaerense, ha sido un entorno importante para la vida de los grupos cazadores recolectores desde el Pleistoceno final y el Holoceno. El análisis de diversas líneas de evidencia permitió conocer y discutir sobre la forma de vida de estos grupos a lo largo del tiempo (Barros y Messineo, 2004; Flegenheimer, Mazzia y Weitzel, 2015; Mazzanti, Martínez y Quintana, 2015; Mazzanti et al., 2013; Mazzia, 2010-2011; Messineo, Kaufmann, Steffan, Favier Dubois y Pal, 2014; Pedrotta, 2013; entre otros). Sin embargo, el sector central de Tandilia, correspondiente al actual partido de Tandil, cuenta con escasas investigaciones que refieran al pasado prehispánico. No obstante, los estudios llevados a cabo allí permiten percibir la importancia y particularidad de la zona para el aprovisionamiento de materias primas líticas a nivel regional (Colombo, 2013; Vecchi, 2010). Este vacío en las investigaciones arqueológicas prehispánicas en Tandil en comparación con el tratamiento de las demás áreas del sistema serrano de Tandilia, sumado a la información aportada por los trabajos sobre localización de fuentes de aprovisionamiento lítico, motivaron el desarrollo de esta tesis.

Como se dijo, el fin último del trabajo fue ampliar y profundizar el conocimiento del pasado pre-contacto del actual partido de Tandil en particular y la región serrana de Tandilia en general, así como también sentar las bases

\footnotetext{
* Área de Arqueología y Antropología, Museo de Ciencias Naturales de Necochea. Av. 10 y 93 s/n (CP 7630), Necochea, Buenos Aires, Argentina. E-mail: arislurselene@gmail.com
}

para futuros trabajos en la zona. Para llevar a cabo el estudio, se propusieron distintos objetivos intermedios que organizaron la tarea: recopilar y sistematizar la información arqueológica del partido de Tandil; estudiar y describir un conjunto de colecciones arqueológicas públicas y/o privadas pertenecientes a la zona de estudio desde una perspectiva relacional; crear un mapeo de potenciales sitios arqueológicos susceptibles de ser prospectados y excavados; analizar los procesos de selección, recolección y guardado que intervinieron en la formación de cada colección; y recopilar las percepciones que subyacen en la comunidad local en relación con el pasado indígena de la zona y el patrimonio arqueológico.

En el marco de esta investigación, se comprende que las personas, tanto en el pasado como en el presente, habitamos paisajes construidos socialmente a partir de vivenciar un conjunto de lugares que están interconectados por las experiencias compartidas con otras personas y con seres no humanos, con las actividades realizadas y con las cosas utilizadas, con los recuerdos y las memorias. En este sentido, habitar no sólo implica estar, sino también sentir, percibir y significar (Hodder, 2012; Ingold, 2000; Mazzia, 2010-2011; Thomas, 2008; Tilley, 2010). Por esto, en este trabajo buscamos reflexionar sobre la interconexión entre las personas (coleccionistas, informantes locales y miembros de instituciones públicas y privadas), con las cosas (las colecciones arqueológicas) y los lugares (los espacios de recolección de las piezas y la percepción de su entorno).

En primer lugar, investigamos sobre la existencia de colecciones públicas y privadas con procedencia conocida y/o registrada dentro del partido y evaluamos las posibilidades de análisis de las mismas. En este sentido, visitamos instituciones públicas y privadas y diseñamos una red de conocidos que nos permitiera generar vínculos con informantes y amateurs locales. Todos los encuentros fueron guiados teórica y metodológicamente por la perspectiva de la entrevista antropológica (Guber, 2004). Los ejes temáticos de las charlas fueron principalmente dos. Por un lado, reflexionar con los coleccionistas locales sobre los procesos particulares de recolección, selección, clasificación y guardado de las piezas que operaron en cada caso. Resultaba pertinente entender si las piezas fueron donadas, regaladas, heredadas o recolectadas por la misma persona; cuáles son los significados construidos y otorgados a las colecciones; por qué fueron conservadas 
y guardadas; dónde y cómo se guardan; cuándo fueron recolectadas; dónde fueron halladas. En definitiva, se buscó reconstruir las historias de vida de cada colección (Pupio, 2005). Por otro lado, indagar sobre las percepciones del pasado indígena de la zona utilizando las colecciones como punto de partida y las relaciones que cada persona construye con las cosas encontradas y recolectadas, con los lugares de hallazgos de las piezas y con otras personas. Por último, visitamos junto a los coleccionistas algunos de los lugares dónde fueron hallados los materiales. El propósito fue georeferenciar los lugares a partir de puntos GPS, para poder plasmar la información en un mapa e indagar sobre las percepciones y valores actuales que las personas tienen en relación con esos lugares.

En total fueron registradas 230 piezas de ocho colecciones arqueológicas, seis privadas y dos públicas, con 14 lugares de procedencia en Tandil. Sin embargo, este número de colecciones relevadas no representa el total del material arqueológico recolectado por coleccionistas locales. A medida que avanzábamos con el proceso de búsqueda de informantes y materiales surgían nuevos contactos y colecciones. Por eso, decidimos estudiar para esta tesis aquellos materiales que no hayan sido vistos por ningún otro arqueólogo/a. Esta condición deja para futuros trabajos las colecciones privadas del paraje La Numancia, ya que fueron advertidas por el Dr. Colombo en su investigación doctoral (Colombo, 2013).

Todas las piezas fueron relevadas de manera sistemática según criterios tipo-morfológicos (Aschero, 1975, 1983; Vecchi, 2010), materias primas, medidas de los ejes principales, lugar de procedencia y fotografiadas. Toda esta información permitió generar la primera base de datos para Tandil que relaciona material arqueológico de colecciones con lugares específicos del entorno serrano. Asimismo, comprendimos que las historias de conformación de las colecciones están íntimamente relacionadas con las representaciones del pasado local, los conocimientos de cada coleccionista y las redes de vínculos que cada persona tiene con otras personas y con los lugares. Pero los lugares significan más que meros puntos de procedencia o hallazgo de materiales. Los lugares fueron y son espacios vivenciados, tanto en el pasado como en el presente. Las personas que descartaron o simplemente dejaron las cosas que luego fueron recolectadas por otras personas y hoy forman parte de las colecciones registradas, habitaron el entorno del partido de Tandil. No se sabe aún de qué manera, hace cuánto, qué actividades hicieron, qué materias primas preferían, pero sí que recorrieron este paisaje serrano.

Entre los distintos puntos conocidos por los relatos y las visitas, El Picadero-Huicha Cura se destaca por ser, en el estado actual de las investigaciones en Tandil, el único sitio con material tallado in situ de ortocuarcita del Grupo Sierras Bayas tanto blanca como coloreada. Por esto, creemos importante continuar con los trabajos de campo allí.

Además, el relevamiento y análisis de las colecciones arqueológicas, el registro escrito y auditivo de las entrevistas y las visitas a los lugares nos permitió discutir y reflexionar sobre las representaciones del pasado indígena local que circulan entre los entrevistados y las instituciones visitadas. La importancia de interpretar las percepciones sobre el pasado indígena local radicó, principalmente, en dos cuestiones. En primer lugar, acceder a ese conocimiento permite reflexionar sobre el rol de los arqueólogos en los procesos de socialización y divulgación de las investigaciones. No es menor el pedido explícito de entes municipales, privados e historiadores en relación con la facilitación de información sobre los trabajos arqueológicos realizados en la zona y los resultados sobre el pasado prehispánico local. A su vez, entender cuáles son las ideas, representaciones, percepciones y necesidades que circulan entre los actores tandilenses entrevistados, permitió generar un primer conocimiento desde el cual realizar futuros trabajos multidimensionales que impliquen la participación activa de la comunidad en los procesos de visibilización e incorporación colectiva del patrimonio arqueológico. Además, se hizo visible que la mayoría de los sujetos entrevistados reconocen una corta profundidad temporal en la historia local. Si bien manifiestan conocer un pasado indígena, éste está relacionado con los momentos post-conquista y los discursos hegemónicos vinculados con la conformación del Estado-Nación (Mazzanti, 2010; Salerno, 2013). Sin embargo, este pasado es narrado por ellos como una historia que representa la vida de un otro lejano en el tiempo y sin continuidad con el presente.

Por último, pudimos pensar y problematizar el rol del amateurismo en Tandil en relación con la práctica arqueológica y los procesos de visibilización del patrimonio cultural, para así reflexionar sobre los procesos de socialización y construcción del conocimiento a nivel local (Pupio, 2011). Estos actores según sus propios criterios y conocimientos llevaron adelante no sólo las tareas de recolección y formación de colecciones, sino también trabajos de investigación, experimentación y divulgación. Por esto, no se pueden dejar de lado al momento de revisar la historia de la construcción del conocimiento arqueológico en Tandil.

\section{Referencias citadas}

》 Achilli, E. L. (2005). Un enfoque antropológico relacional. Algunos núcleos identificatorios. En Achilli, E. L (Ed.), Investigar en Antropología Social. Los Desafíos de Transmitir un Oficio (pp. 15-28). Rosario: Laborde Libros. 
» Aschero, C. (1975). Ensayo para una Clasificación Morfológica de Artefactos Líticos Aplicada a Estudios Tipológicos Comparativos. Informe al CONICET. Manuscrito inédito.

»Aschero, C. (1983). Ensayo para una Clasificación Morfológica de Artefactos Líticos. Apéndices A y B. Cátedra de Ergología y Tecnología. Universidad de Buenos Aires. Manuscrito inédito.

» Barros, M. P. y Messineo, P. (2004). Identificación y aprovisionamiento de ftanita o chert en la cuenca superior del Arroyo Tapalqué (Olavarría, Provincia de Buenos Aires, Argentina). Estudios Atacameños, 28, 87-103.

"Colombo, M. (2013). Los cazadores y recolectores pampeanos y sus rocas. La obtención de materias primas líticas vista desde las canteras arqueológicas del centro de Tandilia. (Tesis Doctoral inédita), Universidad Nacional de La Plata, Argentina.

» Flegenheimer, N., Mazzia, N. y Weitzel, C. (2015). Landscape and rocks in the East-Central portion of the Tandilia Range (Buenos Aires Province, Argentina). PaleoAmerica, 1(2), 163-18o.

» Guber, R. (2004). El salvaje Metropolitano. Reconstrucción del Conocimiento Social en el Trabajo de Campo. Buenos Aires: Paidós.

" Hodder, I. (2012). Entangled. An Archaeology of the Relationships Between Humans and Things. West Sussex: Wiley-Blackwell.

» Ingold, T. (2000). The Perception of the Environment. Essays on Livelihood, Dwelling and Skill. Londres: Routledge.

» Mazzanti, D. (2010). Factores dominantes en el desarrollo de la arqueología pampeana del período posconquista. En J. Nastri y L. M. Ferreira (Eds.), Historias de la Arqueología Sudamericana (pp. 189-210). Buenos Aires: Fundación de Historia Natural Félix de Azara.

» Mazzanti, D. L., Martínez, G., Colobig, M. M., Zucol, A., Passeggi, E., Brea, M., Bonnat, G., Hassan, G., Soria, J. L., Vera, J. A. y Quintana, C. (2013). Avances en los estudios arqueológicos, geoarqueológicos y paleoambientales en las sierras de Tandilia. Resultados preliminares en Alero El Mirador y Abrigo La Grieta. Revista del Museo de La Plata, Sección Antropología, 13(87), 59-76.

» Mazzanti, D. L., Martinez, G. y Quintana, C. (2015). Asentamientos del Holoceno medio en Tandilia oriental. Aportes para el conocimiento de la dinámica poblacional de la región pampeana, Argentina. Relaciones de la Sociedad Argentina de Antropologíal, $\mathrm{XL}(1), 209-231$.

» Mazzia, N. (2010-2011). Lugares y paisajes de cazadoresrecolectores en la pampa bonaerense: cambios y continuidades durante el Pleistoceno final-Holoceno. (Tesis Doctoral inédita), Universidad Nacional de La Plata, Argentina.

» Messineo, P., Kaufmann, C. A., Steffan, P. G., Favier Dubois, C. y Pal, N. (2014). Ocupaciones humanas en un valle interserrano del sector noroccidental de Tandilia: sitio El Puente (partido de Olavarría, Buenos Aires). Relaciones de la Sociedad Argentina de Antropología, $\operatorname{XXXIX(2),~435-462.~}$

»Pedrotta, V. (2013). Reandando los caminos al Chapaleofú: Viejas y nuevas hipótesis sobre las construcciones de piedra del Sistema de Tandilia. Memoria Americana. Cuadernos de Etnohistoria, 21, 269-295.

»Pupio, M. A. (2005). Coleccionistas de objetos históricos, arqueológicos y de ciencias naturales en museos municipales de la provincia de Buenos Aires en la década de 1950. História, Ciências, Saúde - Manguinhos, 12, 205-229.

»Pupio, M. A. (2011). Coleccionistas, aficionados y arqueólogos en la conformación de las colecciones arqueológicas del Museo de La Plata, Argentina (19301950). En M. M. Lopes y A. Heizer (Eds.), Coleccionismos, Prácticas de Campo e Representações (pp. 269-280). Paraíba: Eduepb.

»Salerno, V. M. (2013). Trabajo arqueológico y representaciones del pasado en la provincia de Buenos Aires. Buenos Aires: Facultad de Filosofía y Letras, UBA.

» Thomas, J. (2008). Archaeology, landscapes and dwelling. En B. Bavia y J. Thomas (Eds.), Handbook of Landscape Archaeology (pp. 300-306). Walmut Creek: Left Coast Press.

» Tilley, C. (2010). Interpreting Landscapes. Geologies, Topographies, Identities. Explorations in Landscape Phenomenology 3. Londres: Left Coast Press.

"Vecchi, R. (2010). Bolas de boleadora en los grupos cazadores recolectores de la pampa bonaerense. (Tesis Doctoral inédita), Universidad de Buenos Aires, Argentina. 